\title{
A posteriori error estimates of mixed finite element methods for general optimal control problems governed by integro-differential equations
}

\section{Zuliang Lu $\mathrm{Lu}^{1,2^{*}}$ and Dayong Liu ${ }^{3}$}

*Correspondence:

zulianglux@126.com

'School of Mathematics and

Statistics, Chongqing Three Gorges

University, Chongqing, 404000, P.R.

China

${ }^{2}$ College of Civil Engineering and

Mechanics, Xiangtan University,

Xiangtan, 411105, P.R. China

Full list of author information is

available at the end of the article

\begin{abstract}
In this paper, we study the mixed finite element methods for general convex optimal control problems governed by integro-differential equations. The state and the co-state are discretized by the lowest order Raviart-Thomas mixed finite element spaces and the control is discretized by piecewise constant elements. We derive $a$ posteriori error estimates for the coupled state and control approximation. Such estimates are obtained for some model problems which frequently appear in many applications.
\end{abstract}

MSC: 49J20;65N30

Keywords: optimal control problems; integro-differential equations; mixed finite element methods; a posteriori error estimates

\section{Introduction}

The finite element discretization of optimal control problems has been extensively investigated in early literature. There are two early papers on the numerical approximation of linear quadratic elliptic optimal control problems by Falk [1] and Geveci [2]. In [3], the authors derived a posteriori error estimators for a class of distributed elliptic optimal control problems. These error estimators are shown to be useful in adaptive finite element approximation for the optimal control problems and are implemented in the adaptive approach. Brunner and Yan [4] discussed finite element Galerkin discretization of a class of constrained optimal control problems governed by integral equations and integro-differential equations. The analysis focuses on the derivation of a priori error estimates and a posteriori error estimators for the approximation schemes. Systematic introduction of the finite element method for optimal control problems can be found in [5-7]. Some of the techniques directly relevant to our work can be found in $[8,9]$.

In many control problems, the objective functional contains the gradient of the state variables. Thus, the accuracy of the gradient is important in numerical discretization of the coupled state equations. Mixed finite element methods are appropriate for the state equations in such cases since both the scalar variable and its flux variable can be approximated to the same accuracy by using such methods. Some specialists have made many important works on some topic of mixed finite element methods for linear optimal con-

(c) 2013 Lu and Liu; licensee Springer. This is an Open Access article distributed under the terms of the Creative Commons Attribution License (http://creativecommons.org/licenses/by/2.0), which permits unrestricted use, distribution, and reproduction in any medium, provided the original work is properly cited. 
trol problems. Some realistic regularity assumptions are presented and applied to error estimation by using an operator interpolation. The authors derive $L^{2}$-superconvergence properties for the flux functions along the Gauss lines and for the scalar functions at the Gauss points via mixed projections in [10-12]. Also, $L^{\infty}$-error estimates for general optimal control problems using mixed finite element methods are considered in $[13,14]$. In [15, 16], a posteriori error estimates of mixed finite element methods for general convex optimal control problems are addressed. However, there does not seem to exist much work on theoretical analysis for mixed finite element approximation of optimal control problems governed by integro-differential equations in the literature.

In this paper we derive a posteriori error estimates of mixed finite element methods for general optimal control problems governed by integro-differential equations. We are concerned with the following optimal control problems:

$$
\min _{u \in K \subset U}\left\{g_{1}(\mathbf{p})+g_{2}(y)+j(u)\right\}
$$

subject to the state equation

$$
-\operatorname{div}(A \nabla y)+\int_{\Omega} G(s, t) y(s) d s=f+B u, \quad x \in \Omega,
$$

with the boundary condition

$$
y=0, \quad x \in \partial \Omega,
$$

which can be written in the form of the first-order system

$$
\begin{aligned}
& \operatorname{div} \mathbf{p}+\int_{\Omega} G(s, t) y(s) d s=f+B u, \quad x \in \Omega, \\
& \mathbf{p}=-A \nabla y, \quad x \in \Omega \\
& y=0, \quad x \in \partial \Omega
\end{aligned}
$$

where $\Omega \subset \mathbb{R}^{2}$ is a regular bounded and convex open set with the boundary $\partial \Omega, \Omega_{U}$ is a bounded open set in $\mathbb{R}^{2}$ with the Lipschitz boundary $\partial \Omega_{U}, g_{1}, g_{2}$, and $j$ are convex functionals and $K$ is a closed convex set in $U=L^{2}\left(\Omega_{U}\right)$. Here, $f \in L^{2}(\Omega)$ and $B$ is a continuous linear operator from $L^{2}\left(\Omega_{U}\right)$ to $L^{2}(\Omega), G(\cdot, \cdot) \in H^{1}(\Omega \times \Omega)$, and there are constants $c_{0}, C_{0}>0$ satisfying

$$
c_{0} \leq G(s, t) \leq C_{0}, \quad \forall s, t \in \Omega
$$

The coefficient matrix $A(x)=\left(a_{i, j}(x)\right)_{2 \times 2} \in L^{\infty}\left(\Omega ; \mathbb{R}^{2 \times 2}\right)$ is a symmetric $2 \times 2$-matrix and there are constants $c_{1}, c_{2}>0$ satisfying, for any vector $\mathbf{X} \in \mathbb{R}^{2}, c_{1}\|\mathbf{X}\|_{\mathbb{R}^{2}}^{2} \leq \mathbf{X}^{t} A \mathbf{X} \leq c_{2}\|\mathbf{X}\|_{\mathbb{R}^{2}}^{2}$.

We adopt the standard notation $W^{m, p}(\Omega)$ for Sobolev spaces on $\Omega$ with a norm $\|\cdot\|_{m, p}$ given by $\|v\|_{m, p}^{p}=\sum_{|\alpha| \leq m}\left\|D^{\alpha} v\right\|_{L^{p}(\Omega)}^{p}$, a semi-norm $|\cdot|_{m, p}$ given by $|v|_{m, p}^{p}=$ $\sum_{|\alpha|=m}\left\|D^{\alpha} v\right\|_{L^{p}(\Omega)}^{p}$. We set $W_{0}^{m, p}(\Omega)=\left\{v \in W^{m, p}(\Omega):\left.v\right|_{\partial \Omega}=0\right\}$. For $p=2$, we denote $H^{m}(\Omega)=W^{m, 2}(\Omega), H_{0}^{m}(\Omega)=W_{0}^{m, 2}(\Omega)$, and $\|\cdot\|_{m}=\|\cdot\|_{m, 2},\|\cdot\|=\|\cdot\|_{0,2}$.

Now, we recall a result from Kress [17]. 
Lemma 1.1 We assume that $G(\cdot, \cdot)$ is such that the equations

$$
\begin{aligned}
& -\operatorname{div}(A \nabla \xi)+\int_{\Omega} G(s, t) \xi(t) d t=F_{1} \quad \text { in } \Omega,\left.\xi\right|_{\partial \Omega}=0 \\
& -\operatorname{div}(A \nabla \zeta)+\int_{\Omega} G(s, t) \zeta(s) d s=F_{2} \quad \text { in } \Omega,\left.\zeta\right|_{\partial \Omega}=0
\end{aligned}
$$

have unique solutions $\xi, \zeta \in H^{1}(\Omega)$ for any $F_{1}, F_{2} \in L^{2}(\Omega)$, respectively. Moreover, there exists a positive constant $C$ such that

$$
\begin{aligned}
\|\xi\|_{H^{2}(\Omega)} & \leq C\left\|F_{1}\right\|_{L^{2}(\Omega)}, \\
\|\zeta\|_{H^{2}(\Omega)} & \leq C\left\|F_{2}\right\|_{L^{2}(\Omega)} .
\end{aligned}
$$

In particular, it can be proved that [18] there exist unique solutions for the above integraldifferential equations if $|G(s, t)| \leq a_{0}$, where $a_{0}$ is small enough such that

$$
\int_{\Omega} A \nabla v \nabla v \geq\left(a_{0}+\delta\right)|\Omega|\|v\|_{1, \Omega}^{2}, \quad \forall v \in H^{1}(\Omega)
$$

where $|\Omega|=\int_{\Omega} 1$.

The outline of this paper is as follows. In the next section, we construct the mixed finite element discretization for the optimal control problems governed by integro-differential equations and briefly state the definitions and properties of some interpolation operators. Then we discuss a posteriori error estimates for the intermediate error in Section 3. In Section 4, we derive a posteriori error estimates for the control and state approximations. Finally, some applications are presented in Section 5.

\section{Mixed methods for optimal control problems}

In this section we briefly discuss the mixed finite element discretization of convex optimal control problems (1.1)-(1.3). Let

$$
\mathbf{V}=H(\operatorname{div} ; \Omega)=\left\{\mathbf{v} \in\left(L^{2}(\Omega)\right)^{2}, \operatorname{div} \mathbf{v} \in L^{2}(\Omega)\right\}, \quad W=L^{2}(\Omega) .
$$

The Hilbert space $\mathbf{V}$ is equipped with the following norm:

$$
\|\mathbf{v}\|_{\text {div }}=\|\mathbf{v}\|_{H(\operatorname{div} ; \Omega)}=\left(\|\mathbf{v}\|_{0, \Omega}^{2}+\|\operatorname{div} \mathbf{v}\|_{0, \Omega}^{2}\right)^{1 / 2} .
$$

Then, the weak formulation of the optimal control problems (1.1)-(1.3) is to find (p, $y, u) \in$ $\mathbf{V} \times W \times U$ such that

$$
\begin{aligned}
& \min _{u \in K \subset U}\left\{g_{1}(\mathbf{p})+g_{2}(y)+j(u)\right\}, \\
& \left(A^{-1} \mathbf{p}, \mathbf{v}\right)-(y, \operatorname{div} \mathbf{v})=0, \quad \forall \mathbf{v} \in \mathbf{V}, \\
& (\operatorname{div} \mathbf{p}, w)+\int_{\Omega} \int_{\Omega} G(s, t) y(s) w(t) d s d t=(f+B u, w), \quad \forall w \in W,
\end{aligned}
$$

where the inner product in $L^{2}(\Omega)$ or $\left(L^{2}(\Omega)\right)^{2}$ is denoted by $(\cdot, \cdot)$. It is well known (see, e.g., [19]) that the optimal control problem (2.1)-(2.3) has a unique solution $(\mathbf{p}, y, u)$, and that a 
triplet (p, $y, u)$ is the solution of (2.1)-(2.3) if and only if there is a co-state $(\mathbf{q}, z) \in \mathbf{V} \times W$ such that $(\mathbf{p}, y, \mathbf{q}, z, u)$ satisfies the following optimality conditions:

$$
\begin{aligned}
& \left(A^{-1} \mathbf{p}, \mathbf{v}\right)-(y, \operatorname{div} \mathbf{v})=0, \quad \forall \mathbf{v} \in \mathbf{V}, \\
& (\operatorname{div} \mathbf{p}, w)+\int_{\Omega} \int_{\Omega} G(s, t) y(s) w(t) d s d t=(f+B u, w), \quad \forall w \in W, \\
& \left(A^{-1} \mathbf{q}, \mathbf{v}\right)-(z, \operatorname{div} \mathbf{v})=-\left(g_{1}^{\prime}(\mathbf{p}), \mathbf{v}\right), \quad \forall \mathbf{v} \in \mathbf{V}, \\
& (\operatorname{div} \mathbf{q}, w)+\int_{\Omega} \int_{\Omega} G(s, t) w(s) z(t) d s d t=\left(g_{2}^{\prime}(y), w\right), \quad \forall w \in W, \\
& \left(j^{\prime}(u)+B^{*} z, \tilde{u}-u\right)_{U} \geq 0, \quad \forall \tilde{u} \in K,
\end{aligned}
$$

where $g_{1}^{\prime}, g_{2}^{\prime}$, and $j^{\prime}$ are the derivatives of $g_{1}, g_{2}$, and $j, B^{*}$ is the adjoint operator of $B$, and $(\cdot, \cdot)_{U}$ is the inner product of $U$. In the rest of the paper, we shall simply write the product as $(\cdot, \cdot)$ whenever no confusion should be caused.

We are now able to introduce the discretized problem. To this aim, we consider a family of triangulations or rectangulations $\mathcal{T}_{h}$ of $\bar{\Omega}$. With each element $T \in \mathcal{T}_{h}$, we associate two parameters $\rho(T)$ and $\sigma(T)$, where $\rho(T)$ denotes the diameter of the set $T$ and $\sigma(T)$ is the diameter of the largest ball contained in $T$. The mesh size of the grid is defined by $h=\max _{T \in \mathcal{T}_{h}} \rho(T)$. We suppose that the regularity assumptions are satisfied. There exist two positive constants $\varrho_{1}$ and $\varrho_{2}$ such that

$$
\frac{\rho(T)}{\sigma(T)} \leq \varrho_{1}, \quad \frac{h}{\rho(T)} \leq \varrho_{2}
$$

hold for all $T \in \mathcal{T}_{h}$ and all $h>0$. In addition, $C$ or $c$ denotes a general positive constant independent of $h$.

Let us define $\bar{\Omega}_{h}=\bigcup_{T \in \mathcal{T}_{h}} T$, and let $\Omega_{h}$ and $\Gamma_{h}$ denote its interior and its boundary, respectively. We assume that $\bar{\Omega}_{h}$ is convex and the vertices of $\mathcal{T}_{h}$ placed on the boundary of $\Gamma_{h}$ are points of $\partial \Omega$. We also assume that $\left|\Omega \backslash \Omega_{h}\right| \leq C h^{2}$.

Similarly, we assume that $\mathcal{T}_{h}\left(\Omega_{U}\right)$ are triangulations or rectangulations of $\Omega_{U}$. With each element $s \in \mathcal{T}_{h}\left(\Omega_{U}\right)$, the two parameters $\rho(s)$ and $\sigma(s)$ are assumed to satisfy the regularity assumptions. Next, to every boundary triangle or rectangle $T(s)$ of $\mathcal{T}_{h}\left(\mathcal{T}_{h}\left(\Omega_{U}\right)\right)$, we associate another triangle or rectangle $\hat{T}(\hat{s})$ with curved boundary. We denote by $\hat{\mathcal{T}}_{h}\left(\hat{\mathcal{T}}_{h}\left(\Omega_{U}\right)\right)$ the union of these curved boundary triangles with interior triangles of $\mathcal{T}_{h}\left(\mathcal{T}_{h}\left(\Omega_{U}\right)\right)$ such that

$$
\bar{\Omega}=\bigcup_{\hat{T} \in \hat{T}_{h}} \hat{T}, \quad \bar{\Omega}_{U}=\bigcup_{\hat{s} \in \hat{\mathcal{T}}_{h}\left(\Omega_{U}\right)} \hat{s} .
$$

Let $\mathbf{V}_{h} \times W_{h} \subset \mathbf{V} \times W$ denote the Raviart-Thomas space [20] of the lowest order associated with the triangulations or rectangulations $\mathcal{T}_{h}$ of $\bar{\Omega}$. $P_{k}$ denotes the space of polynomials of total degree at most $k, Q_{m, n}$ indicates the space of polynomials of degree no more than $m$ and $n$ in $x$ and $y$, respectively. If $T$ is a triangle, $\mathbf{V}(T)=\left\{\mathbf{v} \in P_{0}^{2}(T)+x \cdot P_{0}(T)\right\}$, and if $T$ is a rectangle, $\mathbf{V}(T)=\left\{\mathbf{v} \in Q_{1,0}(T) \times Q_{0,1}(T)\right\}$. We define

$$
\begin{aligned}
& \mathbf{V}_{h}:=\left\{\mathbf{v}_{h} \in \mathbf{V}: \forall T \in \mathcal{T}_{h},\left.\mathbf{v}_{h}\right|_{T} \in \mathbf{V}(T) ; \mathbf{v}_{h}=0, \text { on } \bar{\Omega} \backslash \Omega_{h}\right\}, \\
& W_{h}:=\left\{w_{h} \in W: \forall T \in \mathcal{T}_{h},\left.w_{h}\right|_{T}=\text { constant; } w_{h}=0, \text { on } \bar{\Omega} \backslash \Omega_{h}\right\}
\end{aligned}
$$


Associated with $\hat{\mathcal{T}}_{h}\left(\Omega_{U}\right)$ is another finite dimensional subspace $U_{h}$ of $U$ :

$$
U_{h}:=\left\{\tilde{u}_{h} \in U: \forall \hat{s} \in \hat{\mathcal{T}}_{h}\left(\Omega_{U}\right),\left.\tilde{u}_{h}\right|_{\hat{s}}=\text { constant }\right\}
$$

The mixed finite element discretization of (2.1)-(2.3) is as follows: compute $\left(\mathbf{p}_{h}, y_{h}, u_{h}\right) \in$ $\mathbf{V}_{h} \times W_{h} \times U_{h}$ such that

$$
\begin{aligned}
& \min _{u_{h} \in K_{h} \subset U_{h}}\left\{g_{1}\left(\mathbf{p}_{h}\right)+g_{2}\left(y_{h}\right)+j\left(u_{h}\right)\right\}, \\
& \left(A^{-1} \mathbf{p}_{h}, \mathbf{v}_{h}\right)-\left(y_{h}, \operatorname{div} \mathbf{v}_{h}\right)=0, \quad \forall \mathbf{v}_{h} \in \mathbf{V}_{h}, \\
& \left(\operatorname{div} \mathbf{p}_{h}, w_{h}\right)+\int_{\Omega} \int_{\Omega} G(s, t) y_{h}(s) w_{h}(t) d s d t=\left(f+B u_{h}, w_{h}\right), \quad \forall w_{h} \in W_{h},
\end{aligned}
$$

where $K_{h}=U_{h} \cap K$. Under our assumptions on the kernel $G(\cdot, \cdot)$, it can be shown that there exists an $\bar{h}>0$ such that for $h \in(0, \bar{h})$, the mixed finite element approximation

$$
\begin{aligned}
& \left(A^{-1} \mathbf{p}_{h}, \mathbf{v}_{h}\right)-\left(y_{h}, \operatorname{div} \mathbf{v}_{h}\right)=0, \quad \forall \mathbf{v}_{h} \in \mathbf{V}_{h}, \\
& \left(\operatorname{div} \mathbf{p}_{h}, w_{h}\right)+\int_{\Omega} \int_{\Omega} G(s, t) y_{h}(s) w_{h}(t) d s d t=\left(F, w_{h}\right), \quad \forall w_{h} \in W_{h}
\end{aligned}
$$

has a unique solution $\left(\mathbf{p}_{h}, y_{h}\right)$ for any $F \in L^{2}(\Omega)$.

The optimal control problem (2.9)-(2.11) again has a unique solution $\left(\mathbf{p}_{h}, y_{h}, u_{h}\right)$, and a triplet $\left(\mathbf{p}_{h}, y_{h}, u_{h}\right)$ is the solution of (2.9)-(2.11) if and only if there is a co-state $\left(\mathbf{q}_{h}, z_{h}\right) \in$ $\mathbf{V}_{h} \times W_{h}$ such that $\left(\mathbf{p}_{h}, y_{h}, \mathbf{q}_{h}, z_{h}, u_{h}\right)$ satisfies the following optimality conditions:

$$
\begin{aligned}
& \left(A^{-1} \mathbf{p}_{h}, \mathbf{v}_{h}\right)-\left(y_{h}, \operatorname{div} \mathbf{v}_{h}\right)=0, \quad \forall \mathbf{v}_{h} \in \mathbf{V}_{h}, \\
& \left(\operatorname{div} \mathbf{p}_{h}, w_{h}\right)+\int_{\Omega} \int_{\Omega} G(s, t) y_{h}(s) w_{h}(t) d s d t=\left(f+B u_{h}, w_{h}\right), \quad \forall w_{h} \in W_{h}, \\
& \left(A^{-1} \mathbf{q}_{h}, \mathbf{v}_{h}\right)-\left(z_{h}, \operatorname{div} \mathbf{v}_{h}\right)=-\left(g_{1}^{\prime}\left(\mathbf{p}_{h}\right), \mathbf{v}_{h}\right), \quad \forall \mathbf{v}_{h} \in \mathbf{V}_{h}, \\
& \left(\operatorname{div} \mathbf{q}_{h}, w_{h}\right)+\int_{\Omega} \int_{\Omega} G(s, t) w_{h}(s) z_{h}(t) d s d t=\left(g_{2}^{\prime}\left(y_{h}\right), w_{h}\right), \quad \forall w_{h} \in W_{h}, \\
& \left(j^{\prime}\left(u_{h}\right)+B^{*} z_{h}, \tilde{u}_{h}-u_{h}\right)_{U} \geq 0, \quad \forall \tilde{u}_{h} \in K_{h} .
\end{aligned}
$$

In the rest of the paper, we shall use some intermediate variables. For any control function $\tilde{u} \in K$, we first define the state solution $(\mathbf{p}(\tilde{u}), y(\tilde{u}), \mathbf{q}(\tilde{u}), z(\tilde{u}))$ associated with $\tilde{u}$ that satisfies

$$
\begin{aligned}
& \left(A^{-1} \mathbf{p}(\tilde{u}), \mathbf{v}\right)-(y(\tilde{u}), \operatorname{div} \mathbf{v})=0, \quad \forall \mathbf{v} \in \mathbf{V}, \\
& (\operatorname{div} \mathbf{p}(\tilde{u}), w)+\int_{\Omega} \int_{\Omega} G(s, t) y(\tilde{u})(s) w(t) d s d t=(f+B \tilde{u}, w), \quad \forall w \in W \\
& \left(A^{-1} \mathbf{q}(\tilde{u}), \mathbf{v}\right)-(z(\tilde{u}), \operatorname{div} \mathbf{v})=-\left(g_{1}^{\prime}(\mathbf{p}(\tilde{u})), \mathbf{v}\right), \quad \forall \mathbf{v} \in \mathbf{V}, \\
& (\operatorname{div} \mathbf{q}(\tilde{u}), w)+\int_{\Omega} \int_{\Omega} G(s, t) w_{h}(s) z_{h}(\tilde{u})(t) d s d t=\left(g_{2}^{\prime}(y(\tilde{u})), w\right), \quad \forall w \in W
\end{aligned}
$$


Correspondingly, we define the discrete state solution $\left(\mathbf{p}_{h}(\tilde{u}), y_{h}(\tilde{u}), \mathbf{q}_{h}(\tilde{u}), z_{h}(\tilde{u})\right)$ associated with $\tilde{u} \in K$ that satisfies

$$
\begin{aligned}
& \left(A^{-1} \mathbf{p}_{h}(\tilde{u}), \mathbf{v}_{h}\right)-\left(y_{h}(\tilde{u}), \operatorname{div} \mathbf{v}_{h}\right)=0, \quad \forall \mathbf{v}_{h} \in \mathbf{V}_{h}, \\
& \left(\operatorname{div} \mathbf{p}_{h}(\tilde{u}), w_{h}\right)+\int_{\Omega} \int_{\Omega} G(s, t) y_{h}(\tilde{u})(s) w_{h}(t) d s d t=\left(f+B \tilde{u}, w_{h}\right), \quad \forall w_{h} \in W_{h}, \\
& \left(A^{-1} \mathbf{q}_{h}(\tilde{u}), \mathbf{v}_{h}\right)-\left(z_{h}(\tilde{u}), \operatorname{div} \mathbf{v}_{h}\right)=-\left(g_{1}^{\prime}\left(\mathbf{p}_{h}(\tilde{u})\right), \mathbf{v}_{h}\right), \quad \forall \mathbf{v}_{h} \in \mathbf{V}_{h}, \\
& \left(\operatorname{div} \mathbf{q}_{h}(\tilde{u}), w_{h}\right)+\int_{\Omega} \int_{\Omega} G(s, t) w_{h}(s) z_{h}(\tilde{u})(t) d s d t=\left(g_{2}^{\prime}\left(y_{h}(\tilde{u})\right), w_{h}\right), \\
& \quad \forall w_{h} \in W_{h} .
\end{aligned}
$$

Thus, as we defined, the exact solution and its approximation can be written in the following way:

$$
\begin{aligned}
& (\mathbf{p}, y, \mathbf{q}, z)=(\mathbf{p}(u), y(u), \mathbf{q}(u), z(u)), \\
& \left(\mathbf{p}_{h}, y_{h}, \mathbf{q}_{h}, z_{h}\right)=\left(\mathbf{p}_{h}\left(u_{h}\right), y_{h}\left(u_{h}\right), \mathbf{q}_{h}\left(u_{h}\right), z_{h}\left(u_{h}\right)\right) .
\end{aligned}
$$

Let $\varepsilon_{h}$ denote the set of element sides in $\mathcal{T}_{h}$. If there is no risk of confusion, the local mesh size $h$ is defined on both $\mathcal{T}_{h}$ and $\varepsilon_{h}$ by $\left.h\right|_{T}:=h_{T}$ for $T \in \mathcal{T}_{h}$ and $\left.h\right|_{E}:=h_{E}$ for $E \in \mathcal{E}_{h}$, respectively. For all $E \in \mathcal{E}_{h}$, we fix one direction of a unit normal on $E$ pointing in the outside of $\Omega$ in case $E \subset \partial \Omega$. We define that an operator $[v]: H^{1}\left(\mathcal{T}_{h}\right) \rightarrow L^{2}\left(\mathcal{E}_{h}\right)$ is the jump of the function $v$ across the edge $E$, and $\mathbf{t}$ is the tangential unit vector along $E$.

We define $S^{0}\left(\mathcal{T}_{h}\right) \subset L^{2}(\Omega)$ as the piecewise constant space and $S^{1}\left(\mathcal{T}_{h}\right) \subset H^{1}(\Omega)$ or $S_{0}^{1}\left(\mathcal{T}_{h}\right) \subset H_{0}^{1}(\Omega)$ as continuous and piecewise linear functions, piecewise is understood with respect to $\mathcal{T}_{h}$. We consider Clement's interpolation operator $I_{h}: H^{1}(\Omega) \rightarrow S^{1}\left(\mathcal{T}_{h}\right)$ which satisfies [21]

$$
\begin{array}{ll}
\left\|v-I_{h} v\right\|_{0, T} \leq C h_{T}\|v\|_{1, w_{T}}, & \forall v \in H_{0}^{1}(\Omega), \\
\left\|v-I_{h} v\right\|_{0, E} \leq C h_{E}^{1 / 2}\|v\|_{1, w_{E}}, & \forall v \in H_{0}^{1}(\Omega)
\end{array}
$$

for each $T \in \mathcal{T}_{h}$ and $E \in \mathcal{E}_{h}, w_{T}=\left\{T^{\prime} \in \mathcal{T}_{h}, \bar{T} \cap \bar{T}^{\prime} \neq \emptyset\right\}, w_{E}=\left\{T \in \mathcal{T}_{h}, E \in \bar{T}\right\}$.

Now, we define the standard $L^{2}(\Omega)$-orthogonal projection $P_{h}: W \rightarrow W_{h}$, which satisfies the approximation property [22]:

$$
\left\|h^{-1} \cdot\left(v-P_{h} v\right)\right\|_{0} \leq C\left\|\nabla_{h} v\right\|_{0}, \quad \forall \mathbf{v} \in H^{1}\left(\mathcal{T}_{h}\right) .
$$

Let us define the interpolation operator $\Pi_{h}: \mathbf{V} \rightarrow \mathbf{V}_{h}$, which satisfies: for any $\mathbf{q} \in \mathbf{V}$,

$$
\int_{T}\left(\mathbf{q}-\Pi_{h} \mathbf{q}\right) \cdot \mathbf{v}_{h} d x d y=0, \quad \forall \mathbf{v}_{h} \in \mathbf{V}_{h}, T \in \mathcal{T}_{h}
$$

We have the commuting diagram property

$$
\operatorname{div} \circ \Pi_{h}=P_{h} \circ \operatorname{div}: \mathbf{V} \rightarrow W_{h} \text { and } \operatorname{div}\left(I-\Pi_{h}\right) \mathbf{V} \perp W_{h},
$$

where and after, $I$ denotes an identity operator. 
Next, the interpolation operator $\Pi_{h}$ satisfies the local error estimate

$$
\left\|h^{-1} \cdot\left(\mathbf{q}-\Pi_{h} \mathbf{q}\right)\right\|_{0} \leq C|\mathbf{q}|_{1, \mathcal{T}_{h}}, \quad \mathbf{q} \in H^{1}\left(\mathcal{T}_{h}\right) \cap \mathbf{V} .
$$

Furthermore, we assume that [23]

$$
\begin{aligned}
& W_{h} \subset H^{1}\left(\mathcal{T}_{h}\right),\left.\quad A^{-1} p_{h}\right|_{T} \in P_{l},\left.\quad \nabla_{h} y_{h}\right|_{T} \in P_{l}, \quad \forall T \in \mathcal{T}_{h}, \\
& S^{0}\left(\mathcal{T}_{h}\right)^{2} \cap H^{1}(\operatorname{div} ; \Omega) \subset \mathbf{V}_{h} \subset H^{1}\left(\mathcal{T}_{h}\right) \cap H^{1}(\operatorname{div} ; \Omega) .
\end{aligned}
$$

\section{A posteriori error estimates for the intermediate errors}

Given $u \in K$, let $S_{1}, S_{2}$ be the inverse operators of state equation (2.3) such that $\mathbf{p}(u)=$ $S_{1} B u$ and $y(u)=S_{2} B u$ are the solutions of state equation (2.3). Similarly, for given $u_{h} \in K_{h}$, $\mathbf{p}_{h}\left(u_{h}\right)=S_{1 h} B u_{h}, y_{h}\left(u_{h}\right)=S_{2 h} B u_{h}$ are the solutions of discrete state equation (2.11). Let

$$
\begin{aligned}
& J(u)=g_{1}\left(S_{1} B u\right)+g_{2}\left(S_{2} B u\right)+j(u), \\
& J_{h}\left(u_{h}\right)=g_{1}\left(S_{1 h} B u_{h}\right)+g_{2}\left(S_{2 h} B u_{h}\right)+j\left(u_{h}\right) .
\end{aligned}
$$

It is clear that $J$ and $J_{h}$ are well defined and continuous on $K$ and $K_{h}$. Also, the functional $J_{h}$ can be naturally extended on $K$. Then (2.1) and (2.9) can be represented as

$$
\begin{aligned}
& \min _{u \in K}\{J(u)\}, \\
& \min _{u_{h} \in K_{h}}\left\{J_{h}\left(u_{h}\right)\right\} .
\end{aligned}
$$

An additional assumption is needed. We assume that the cost function $J$ is strictly convex near the solution $u$, i.e., for the solution $u$, there exists a neighborhood of $u$ in $L^{2}$ such that $J$ is convex in the sense that there is a constant $c>0$ satisfying

$$
\left(J^{\prime}(u)-J^{\prime}(v), u-v\right) \geq c\|u-v\|_{U}^{2},
$$

for all $v$ in this neighborhood of $u$. The convexity of $J(\cdot)$ is closely related to the secondorder sufficient optimality conditions of optimal control problems, which are assumed in many studies on numerical methods of the problem. For instance, in many references, the authors assume the following second-order sufficiently optimality condition (see [21, 24]): there is $c>0$ such that $J^{\prime \prime}(u) v^{2} \geq c\|v\|_{0}^{2}$.

Now, we are able to derive the main result.

Lemma 3.1 Let $u$ and $u_{h}$ be the solutions of (3.1) and (3.2), respectively. Assume that $K_{h} \subset K$. In addition, assume that $\left.\left(J_{h}^{\prime}\left(u_{h}\right)\right)\right|_{s} \in H^{1}(s), \forall s \in \mathcal{T}_{h}\left(\Omega_{U}\right)$, and that there is a $v_{h} \in K_{h}$ such that

$$
\left.\mid J_{h}^{\prime}\left(u_{h}\right), v_{h}-u\right) \mid \leq C \sum_{s \in \mathcal{T}_{h}\left(\Omega_{U}\right)} h_{s}\left\|J_{h}^{\prime}\left(u_{h}\right)\right\|_{H^{1}(s)}\left\|u-u_{h}\right\|_{L^{2}(s)} .
$$

Then we have

$$
\left\|u-u_{h}\right\|_{U}^{2} \leq C \eta_{1}^{2}+C\left\|z\left(u_{h}\right)-z_{h}\right\|_{0}^{2}
$$


where

$$
\eta_{1}^{2}=\sum_{s \in \mathcal{T}_{h}\left(\Omega_{U}\right)} h_{s}^{2}\left\|j^{\prime}\left(u_{h}\right)+B^{*} z_{h}\right\|_{H^{1}(s)}^{2} .
$$

Proof It follows from (3.1) and (3.2) that

$$
\begin{aligned}
& \left(J^{\prime}(u), u-v\right) \leq 0, \quad \forall v \in K, \\
& \left(J_{h}^{\prime}\left(u_{h}\right), u_{h}-v_{h}\right) \leq 0, \quad \forall v_{h} \in K_{h} \subset K .
\end{aligned}
$$

Then it follows from (3.3) and (3.7)-(3.8) that

$$
\begin{aligned}
c\left\|u-u_{h}\right\|_{U}^{2} & \leq\left(J^{\prime}(u)-J^{\prime}\left(u_{h}\right), u-u_{h}\right)_{U} \\
& \leq-\left(J^{\prime}\left(u_{h}\right), u-u_{h}\right)_{U} \\
& =\left(J_{h}^{\prime}\left(u_{h}\right), u_{h}-u\right)_{U}+\left(J_{h}^{\prime}\left(u_{h}\right)-J^{\prime}\left(u_{h}\right), u-u_{h}\right)_{U} \\
& \leq\left(J_{h}^{\prime}\left(u_{h}\right), v_{h}-u\right)_{U}+\left(J_{h}^{\prime}\left(u_{h}\right)-J^{\prime}\left(u_{h}\right), u-u_{h}\right)_{U} .
\end{aligned}
$$

From (3.4), (3.9), and the Schwarz inequality, we get that

$$
\begin{aligned}
c\left\|u-u_{h}\right\|_{U}^{2} \leq & C \sum_{s \in \mathcal{T}_{h}\left(\Omega_{U}\right)} h_{s}\left\|J_{h}^{\prime}\left(u_{h}\right)\right\|_{H^{1}(s)}\left\|u-u_{h}\right\|_{L^{2}(s)} \\
& +C\left\|J_{h}^{\prime}\left(u_{h}\right)-J^{\prime}\left(u_{h}\right)\right\|_{U^{\prime}}\left\|u-u_{h}\right\|_{U} \\
\leq & C \sum_{s \in \mathcal{T}_{h}\left(\Omega_{U}\right)} h_{s}^{2}\left\|J_{h}^{\prime}\left(u_{h}\right)\right\|_{H^{1}(s)}^{2} \\
& +C\left\|J_{h}^{\prime}\left(u_{h}\right)-J^{\prime}\left(u_{h}\right)\right\|_{U}^{2}+\delta\left\|u-u_{h}\right\|_{U}^{2} .
\end{aligned}
$$

It is not difficult to show

$$
J_{h}^{\prime}\left(u_{h}\right)=j^{\prime}\left(u_{h}\right)+B^{*} z_{h}, \quad J^{\prime}\left(u_{h}\right)=j^{\prime}\left(u_{h}\right)+B^{*} z\left(u_{h}\right),
$$

where $z\left(u_{h}\right)$ is the solution of equations (2.19)-(2.22). From (3.11), it is easy to derive

$$
\left\|J_{h}^{\prime}\left(u_{h}\right)-J^{\prime}\left(u_{h}\right)\right\|_{U}=\left\|B^{*}\left(z_{h}-z\left(u_{h}\right)\right)\right\|_{U} \leq C\left\|z_{h}-z\left(u_{h}\right)\right\|_{0} .
$$

It is clear that (3.5) can be derived from (3.10)-(3.12).

Fix a function $u_{h} \in U_{h}$, let $\left(\mathbf{p}\left(u_{h}\right), y\left(u_{h}\right)\right) \in \mathbf{V} \times W$ be the solution of equations (2.19)(2.20). Set some intermediate errors: $\varepsilon_{1}:=\mathbf{p}\left(u_{h}\right)-\mathbf{p}_{h}, e_{1}:=y\left(u_{h}\right)-y_{h}$.

To analyze the fixing $u_{h}$ approach, let us first note the following error equations from (2.10)-(2.11) and (2.19)-(2.20):

$$
\begin{aligned}
& \left(A^{-1} \varepsilon_{1}, \mathbf{v}_{h}\right)-\left(e_{1}, \operatorname{div} \mathbf{v}_{h}\right)=0, \quad \forall \mathbf{v}_{h} \in \mathbf{V}_{h}, \\
& \left(\operatorname{div} \varepsilon_{1}, w_{h}\right)+\int_{\Omega} \int_{\Omega} G(s, t) e_{1}(s) w_{h}(t) d s d t=0, \quad \forall w_{h} \in W_{h} .
\end{aligned}
$$


Lemma 3.2 For the Raviart-Thomas elements, there is a positive constant $C$, which only depends on $A, \Omega$, and the shape of the elements and their maximal polynomial degree $k$, such that

$$
\left\|\mathbf{p}\left(u_{h}\right)-\mathbf{p}_{h}\right\|_{\text {div }}+\left\|y\left(u_{h}\right)-y_{h}\right\|_{0} \leq C \eta_{2}
$$

where

$$
\begin{aligned}
\eta_{2}:= & {\left[\sum _ { T \in \mathcal { T } _ { h } } \left(\left\|f+B u_{h}-\operatorname{div} \mathbf{p}_{h}-\int_{\Omega} G(s, t) y_{h}(s) d s\right\|_{0, T}^{2}+h_{T}^{2} \cdot\left\|\operatorname{curl}_{h}\left(A^{-1} \mathbf{p}_{h}\right)\right\|_{0, T}^{2}\right.\right.} \\
& \left.\left.+h_{T}^{2} \cdot \min _{w_{h} \in W_{h}}\left\|\nabla_{h} w_{h}-A^{-1} \mathbf{p}_{h}\right\|_{0, T}^{2}+\left\|h_{E}^{1 / 2}\left[\left(A^{-1} \mathbf{p}_{h}\right) \cdot \mathbf{t}\right]\right\|_{0, \partial T}^{2}\right)\right]^{1 / 2} \cdot
\end{aligned}
$$

Proof We analyze a Helmholtz decomposition [23] of $A^{-1} \mathbf{p}_{h}$ with a fixing $\varphi \in H_{0}^{1}(\Omega)$ such that $-\operatorname{div}(A \nabla \varphi)=\operatorname{div} \mathbf{p}_{h}$. Then there is some $\psi \in H^{1}(\Omega)$ satisfying $\int_{\Omega} \psi d x=0, \operatorname{Curl} \psi \perp$ $\nabla H_{0}^{1}(\Omega)$ and

$$
\mathbf{p}_{h}=-A \nabla \varphi+\operatorname{Curl} \psi
$$

From (3.17) and (1.8)-(1.11), we derive

$$
\varepsilon_{1}=A \nabla \chi-\operatorname{Curl} \psi \quad \text { with } \chi=\varphi-y\left(u_{h}\right) \in H_{0}^{1}(\Omega)
$$

and hence the error decomposition

$$
\left(A^{-1} \varepsilon_{1}, \varepsilon_{1}\right)=(A \nabla \chi, \nabla \chi)+\left(A^{-1} \operatorname{Curl} \psi, \operatorname{Curl} \psi\right)
$$

It follows from Poincare's inequality and (2.29) that

$$
\begin{aligned}
(A \nabla \chi, \nabla \chi) & =\left(\nabla \chi, \varepsilon_{1}\right)=-\left(\operatorname{div} \varepsilon_{1}, \chi\right) \\
& =\left(\operatorname{div} \varepsilon_{1}, P_{h} \chi-\chi\right)-\left(\operatorname{div} \varepsilon_{1}, P_{h} \chi\right) \\
& \leq C\left\|h_{T} \cdot \operatorname{div} \varepsilon_{1}\right\|_{0} \cdot\left\|A^{1 / 2} \nabla \chi\right\|_{0}+C\left\|\operatorname{div} \varepsilon_{1}\right\|_{0} \cdot\left\|P_{h} \chi\right\|_{0} \\
& \leq C\left\|h_{T} \cdot \operatorname{div} \varepsilon_{1}\right\|_{0} \cdot\left\|A^{1 / 2} \nabla \chi\right\|_{0}+C\left\|\operatorname{div} \varepsilon_{1}\right\|_{0} \cdot\left\|A^{1 / 2} \nabla \chi\right\|_{0} .
\end{aligned}
$$

To estimate the second contribution to the right-hand side of (3.19), we utilize Clement's operator $I_{h}$. Note that $I_{h} \psi \in S^{1}\left(\mathcal{T}_{h}\right) \subset H^{1}(\Omega)$, $\operatorname{Curl} I_{h} \psi \in S^{0}\left(\mathcal{T}_{h}\right)^{2} \cap H^{1}(\operatorname{div} ; \Omega) \subset \mathbf{V}_{h}$ and $\operatorname{Curl} I_{h} \psi \perp \nabla H_{0}^{1}(\Omega)$, whence $\operatorname{div}\left(\operatorname{Curl} I_{h} \psi\right)=0$. Therefore, we obtain

$$
\left(A^{-1} \operatorname{Curl} \psi_{1}, \operatorname{Curl} I_{h} \psi\right)=-\left(A^{-1} \varepsilon_{1}, \operatorname{Curl} I_{h} \psi\right)=-\left(e_{1}, \operatorname{div} \operatorname{Curl} I_{h} \psi\right)=0 .
$$

Utilizing (3.17) and (2.27)-(2.28), we infer

$$
\begin{aligned}
& \left(A^{-1} \operatorname{Curl} \psi, \operatorname{Curl} \psi\right) \\
& \quad=\left(A^{-1} \operatorname{Curl} \psi, \operatorname{Curl}\left(\psi-I_{h} \psi\right)\right)=\left(A^{-1} \mathbf{p}_{h}, \operatorname{Curl}\left(\psi-I_{h} \psi\right)\right)
\end{aligned}
$$




$$
\begin{aligned}
& =-\left(\psi-I_{h} \psi, \operatorname{curl}_{h}\left(A^{-1} \mathbf{p}_{h}\right)\right)+\left(\left[\left(A^{-1} \mathbf{p}_{h}\right) \cdot \mathbf{t}\right], \psi-I_{h} \psi\right)_{\varepsilon_{h}} \\
& \leq C\left(\left\|h_{T} \cdot \operatorname{curl}_{h}\left(A^{-1} \mathbf{p}_{h}\right)\right\|_{0}+\left\|h_{E}^{1 / 2} \cdot\left[\left(A^{-1} \mathbf{p}_{h}\right) \cdot \mathbf{t}\right]\right\|_{0, \varepsilon_{h}}\right)\|\psi\|_{1} .
\end{aligned}
$$

With Poincare's inequality we deduce

$$
\|\psi\|_{1} \leq C\|\nabla \psi\|_{0}=C\|\operatorname{Curl} \psi\|_{0} \leq C\left\|A^{-1 / 2} \operatorname{Curl} \psi\right\|_{0} .
$$

From (2.20), we have

$$
\begin{aligned}
\operatorname{div} \varepsilon_{1} & =f+B u_{h}-\operatorname{div} \mathbf{p}_{h}-\int_{\Omega} G(s, t) y\left(u_{h}\right)(s) d s \\
& =f+B u_{h}-\operatorname{div} \mathbf{p}_{h}-\int_{\Omega} G(s, t) y_{h}(s) d s-\int_{\Omega} G(s, t) e_{1}(s) d s,
\end{aligned}
$$

and together with (3.19)-(3.23) we have

$$
\begin{aligned}
\left\|\varepsilon_{1}\right\|_{\text {div }} \leq & C\left(\left\|f+B u_{h}-\operatorname{div} \mathbf{p}_{h}-\int_{\Omega} G(s, t) y_{h}(s) d s\right\|_{0}+\left\|e_{1}\right\|_{0}\right. \\
& \left.+h_{T} \cdot\left\|\operatorname{curl}_{h}\left(A^{-1} \mathbf{p}_{h}\right)\right\|_{0}+\left\|h_{E}^{1 / 2}\left[\left(A^{-1} \mathbf{p}_{h}\right) \cdot \mathbf{t}\right]\right\|_{0, \varepsilon_{h}}\right) .
\end{aligned}
$$

Now, let us estimate $\left\|e_{1}\right\|_{0}$. Let $\xi$ be the solution of (1.9) with $F_{1}=y\left(u_{h}\right)-y_{h}$. According to (1.8)-(1.11), we have $\xi \in H_{0}^{1}(\Omega) \cap H^{2}\left(\mathcal{T}_{h}\right)$. Then it follows from (1.9), (2.14)-(2.15) and (2.30) that

$$
\begin{aligned}
\left\|e_{1}\right\|_{0}^{2}= & \left(y\left(u_{h}\right)-y_{h},-\operatorname{div}(A \nabla \xi)+\int_{\Omega} G(s, t) \xi(t) d t\right) \\
= & -\left(\mathbf{p}\left(u_{h}\right), \nabla \xi\right)+\left(y_{h}, \operatorname{div} \Pi_{h}(A \nabla \xi)\right)+\left(y\left(u_{h}\right)-y_{h}, \int_{\Omega} G(s, t) \xi(t) d t\right) \\
= & \left(\operatorname{div} \mathbf{p}\left(u_{h}\right), \xi\right)+\int_{\Omega} \int_{\Omega} G(s, t) y\left(u_{h}\right)(s) \xi(t) d s d t \\
& +\left(A^{-1} \mathbf{p}_{h}, \Pi_{h}(A \nabla \xi)\right)-\left(y_{h}, \int_{\Omega} G(s, t) \xi(t) d t\right) \\
= & \left(f+B u_{h}-\operatorname{div} \mathbf{p}_{h}-\int_{\Omega} G(s, t) y_{h}(s) d s, \xi\right)+\left(\nabla_{h} w_{h}-A^{-1} \mathbf{p}_{h},\left(I-\Pi_{h}\right)(A \nabla \xi)\right) \\
\leq & C\left(\left\|f+B u_{h}-\operatorname{div} \mathbf{p}_{h}-\int_{\Omega} G(s, t) y_{h}(s) d s\right\|_{0}+\left\|h \cdot\left(\nabla_{h} w_{h}-A^{-1} \mathbf{p}_{h}\right)\right\|_{0}\right) \cdot\|\xi\|_{2} \\
\leq & C\left(\left\|f+B u_{h}-\operatorname{div} \mathbf{p}_{h}-\int_{\Omega} G(s, t) y_{h}(s) d s\right\|_{0}^{2}+\left\|h \cdot\left(\nabla_{h} w_{h}-A^{-1} \mathbf{p}_{h}\right)\right\|_{0}^{2}\right)+\delta\left\|e_{1}\right\|_{0}^{2}
\end{aligned}
$$

for any $w_{h} \in W_{h}$. Using the triangle inequality, we obtain

$$
\left\|e_{1}\right\|_{0} \leq C\left(\left\|f+B u_{h}-\operatorname{div} \mathbf{p}_{h}-\int_{\Omega} G(s, t) y_{h}(s) d s\right\|_{0}+\left\|h \cdot\left(\nabla_{h} w_{h}-A^{-1} \mathbf{p}_{h}\right)\right\|_{0}\right) .
$$

So, Lemma 3.2 has been proved by combining with (3.24) and (3.25).

Moreover, we can prove the reverse inequality of (3.15). 
Lemma 3.3 For the Raviart-Thomas elements, there is a positive constant $C$, which only depends on $A, \Omega$, and the shape of the elements and their maximal polynomial degree $k$, such that

$$
C \eta_{2} \leq\left\|\mathbf{p}\left(u_{h}\right)-\mathbf{p}_{h}\right\|_{\text {div }}+\left\|y\left(u_{h}\right)-y_{h}\right\|_{0} .
$$

Proof First, from (3.23) we derive that

$$
f+B u_{h}-\operatorname{div} \mathbf{p}_{h}-\int_{\Omega} G(s, t) y_{h}(s) d s=\operatorname{div} \varepsilon_{1}+\int_{\Omega} G(s, t) e_{1}(s) d s,
$$

then we have

$$
\left\|f+B u_{h}-\operatorname{div} \mathbf{p}_{h}-\int_{\Omega} G(s, t) y_{h}(s) d s\right\|_{0, T} \leq C\left(\left\|\varepsilon_{1}\right\|_{H(\mathrm{div} ; T)}+\left\|e_{1}\right\|_{0, T}\right) .
$$

Next, using the standard Bubble function technique, we fix $\varrho_{T} \in P_{3}$ with $0 \leq \varrho_{T} \leq 1=$ $\max \varrho_{T}$ and zero boundary values on $T$ to derive

$$
C\left\|\operatorname{curl}\left(A^{-1} \mathbf{p}_{h}\right)\right\|_{0, T}^{2} \leq\left\|\varrho_{T}^{1 / 2} \cdot \operatorname{curl}\left(A^{-1} \mathbf{p}_{h}\right)\right\|_{0, T}^{2} .
$$

Using (3.17) and (3.18), we obtain

$$
\begin{aligned}
\left\|\varrho_{T}^{1 / 2} \cdot \operatorname{curl}\left(A^{-1} \mathbf{p}_{h}\right)\right\|_{0, T}^{2} & =\int_{T}\left(A^{-1} \varepsilon_{1}\right) \cdot \operatorname{Curl}\left(\varrho_{T}^{1 / 2} \cdot \operatorname{curl}\left(A^{-1} \mathbf{p}_{h}\right)\right) d x \\
& \leq C\left\|A^{-1} \varepsilon_{1}\right\|_{0, T} \cdot\left|\varrho_{T}^{1 / 2} \cdot \operatorname{curl}\left(A^{-1} \mathbf{p}_{h}\right)\right|_{1, T} \\
& \leq C\left\|\varepsilon_{1}\right\|_{H(\mathrm{div} ; T)} \cdot h_{T}^{-1} \cdot\left\|\varrho_{T}^{1 / 2} \cdot \operatorname{curl}\left(A^{-1} \mathbf{p}_{h}\right)\right\|_{0, T}
\end{aligned}
$$

since $\varrho_{T}^{1 / 2} \cdot \operatorname{curl}\left(A^{-1} \mathbf{p}_{h}\right) \in P_{l+2}$ with zero boundary values on $T$. Combining (3.29) and (3.30), we have

$$
h_{T} \cdot\left\|\operatorname{curl}\left(A^{-1} \mathbf{p}_{h}\right)\right\|_{0, T} \leq C\left\|\varepsilon_{1}\right\|_{H(\text { div } ; T)} .
$$

Now, let $\varrho_{E}$ denote the continuous function satisfying $\varrho_{E} \in P_{2}$ with $0 \leq \varrho_{E} \leq 1=\max \varrho_{E}$ on $w_{E}$. Let $\sigma=\left[\left(A^{-1} \mathbf{p}_{h}\right) \cdot \mathbf{t}\right]$. Using continuous extension on the reference element in [25], there exists an extension operator $P: C(E) \rightarrow C\left(w_{E}\right)$ satisfying $\left.P \sigma\right|_{E}=\sigma$ and

$$
c_{1} h_{E}^{1 / 2}\|\sigma\|_{0, E} \leq\left\|\varrho_{E}^{1 / 2} P \sigma\right\|_{0, w_{E}} \leq c_{2} h_{E}^{1 / 2}\|\sigma\|_{0, E},
$$

where $c_{1}$ and $c_{2}$ are positive constants. By the integration by parts formula and (3.31)(3.32), we obtain

$$
\begin{aligned}
C\|\sigma\|_{0, E}^{2} & \leq\left\|\varrho_{E}^{1 / 2} \sigma\right\|_{0, E}^{2}=-\int_{E}\left(\varrho_{E} P \sigma\right) \cdot\left[A^{-1} \varepsilon_{1} \cdot \mathbf{t}\right] d s \\
& =-\int_{w_{E}}\left(A^{-1} \varepsilon_{1}\right) \cdot \operatorname{Curl}\left(\varrho_{E} P \sigma\right) d x-\int_{w_{E}}\left(\varrho_{E} P \sigma\right) \operatorname{curl}\left(A^{-1} \varepsilon_{1}\right) d x \\
& =-\int_{w_{E}}\left(A^{-1} \varepsilon_{1}\right) \cdot \operatorname{Curl}\left(\varrho_{E} P \sigma\right) d x-\int_{w_{E}}\left(\varrho_{E} P \sigma\right) \operatorname{curl}\left(A^{-1} \mathbf{p}_{h}\right) d x
\end{aligned}
$$




$$
\begin{aligned}
& \leq\left\|\varepsilon_{1}\right\|_{0, w_{E}}\left|\varrho_{E} P \sigma\right|_{1, w_{E}}+\left\|\varrho_{E} P \sigma\right\|_{0, w_{E}}\left\|\operatorname{curl}\left(A^{-1} \mathbf{p}_{h}\right)\right\|_{0, w_{E}} \\
& \leq C h_{E}^{-1 / 2}\|\sigma\|_{0, E} \cdot\left\|\varepsilon_{1}\right\|_{H\left(\mathrm{div} ; w_{E}\right)},
\end{aligned}
$$

where the inverse estimates have been used. Then we obtain that

$$
\left\|h^{1 / 2}\left[\left(A^{-1} \mathbf{p}_{h}\right) \cdot \mathbf{t}\right]\right\|_{0, E} \leq C\left\|\varepsilon_{1}\right\|_{H\left(\mathrm{div} ; w_{E}\right)} .
$$

Finally, as in (3.29) and with integration by parts, we derive that

$$
\begin{aligned}
C & \left\|A^{-1} \mathbf{p}_{h}-\nabla_{h} y_{h}\right\|_{0, T}^{2} \\
& \leq\left\|\varrho_{T}^{1 / 2}\left(A^{-1} \mathbf{p}_{h}-\nabla_{h} y_{h}\right)\right\|_{0, T}^{2} \\
& =-\int_{T} \varrho_{T} A^{-1} \varepsilon_{1}\left(A^{-1} \mathbf{p}_{h}-\nabla_{h} y_{h}\right) d x-\int_{T} e_{1} \operatorname{div}\left(\varrho_{T}\left(A^{-1} \mathbf{p}_{h}-\nabla_{h} y_{h}\right)\right) d x \\
& \leq\left\|A^{-1} \varepsilon_{1}\right\|_{0, T}\left\|\varrho_{T}\left(A^{-1} \mathbf{p}_{h}-\nabla_{h} y_{h}\right)\right\|_{0, T}+\left\|e_{1}\right\|_{0, T}\left|\varrho_{T}\left(A^{-1} \mathbf{p}_{h}-\nabla_{h} y_{h}\right)\right|_{1, T} \\
& \leq\left(\left\|A^{-1} \varepsilon_{1}\right\|_{0, T}+\left\|e_{1}\right\|_{0, T} \cdot h_{T}^{-1}\right)\left\|\varrho_{T}\left(A^{-1} \mathbf{p}_{h}-\nabla_{h} y_{h}\right)\right\|_{0, T},
\end{aligned}
$$

where the inverse inequality has been used. From (3.35) it is clear that

$$
h_{T} \min _{w_{h} \in W_{h}}\left\|A^{-1} \mathbf{p}_{h}-\nabla_{h} w_{h}\right\|_{0, T} \leq C\left(\left\|e_{1}\right\|_{0, T}+h_{T}\left\|A^{-1} \varepsilon_{1}\right\|_{0, T}\right)
$$

Then Lemma 3.3 is proved by combining (3.28), (3.31), (3.34) and (3.36).

Arguing as in the proof of Lemma 3.2, we obtain the following results.

Lemma 3.4 For the Raviart-Thomas elements, there is a positive constant $C$, which only depends on $A, \Omega$, and the shape of the elements and their maximal polynomial degree $k$, such that

$$
\left\|\mathbf{q}\left(u_{h}\right)-\mathbf{q}_{h}\right\|_{\text {div }}+\left\|z\left(u_{h}\right)-z_{h}\right\|_{0} \leq C\left(\eta_{2}+\eta_{3}\right)
$$

where

$$
\begin{aligned}
\eta_{3}:= & {\left[\sum _ { T \in \mathcal { T } _ { h } } \left(\left\|g_{2}^{\prime}\left(y_{h}\right)-\operatorname{div} \mathbf{q}_{h}-\int_{\Omega} G(s, t) z_{h}(t) d t\right\|_{0, T}^{2}+h_{T}^{2} \cdot\left\|\operatorname{curl}_{h}\left(A^{-1} \mathbf{q}_{h}+g_{1}^{\prime}\left(\mathbf{p}_{h}\right)\right)\right\|_{0, T}^{2}\right.\right.} \\
& +h_{T}^{2} \cdot \min _{w_{h} \in W_{h}}\left\|\nabla_{h} w_{h}-A^{-1} \mathbf{q}_{h}-g_{1}^{\prime}\left(\mathbf{p}_{h}\right)\right\|_{0, T}^{2} \\
& \left.\left.+\left\|h_{E}^{1 / 2}\left[\left(A^{-1} \mathbf{q}_{h}+g_{1}^{\prime}\left(\mathbf{p}_{h}\right)\right) \cdot \mathbf{t}\right]\right\|_{0, \partial T}^{2}\right)\right]^{1 / 2}
\end{aligned}
$$

Using Lemma 3.1, Lemma 3.2, and Lemma 3.4, we derive the following results.

Theorem 3.1 Let $u$ and $u_{h}$ be the solutions of (3.1) and (3.2), respectively. Assume that $K_{h} \subset K$. In addition, assume that $\left.\left(V_{h}^{\prime}\left(u_{h}\right)\right)\right|_{s} \in H^{s}(s), \forall s \in \mathcal{T}_{h}\left(\Omega_{U}\right)(s=0$ or 1$)$, and that there 
is a $v_{h} \in K_{h}$ such that

$$
\left.\mid J_{h}^{\prime}\left(u_{h}\right), v_{h}-u\right) \mid \leq C \sum_{s \in \mathcal{T}_{h}\left(\Omega_{U}\right)} h_{s}\left\|J_{h}^{\prime}\left(u_{h}\right)\right\|_{H^{1}(s)}\left\|u-u_{h}\right\|_{L^{2}(s)}^{s} .
$$

Then, for the Raviart-Thomas elements, there is a positive constant $C$, which only depends on $A, \Omega$, and the shape of the elements and their maximal polynomial degree $k$, such that

$$
\begin{gathered}
\left\|\mathbf{p}\left(u_{h}\right)-\mathbf{p}_{h}\right\|_{\mathrm{div}}^{2}+\left\|y\left(u_{h}\right)-y_{h}\right\|_{0}^{2}+\left\|\mathbf{q}\left(u_{h}\right)-\mathbf{q}_{h}\right\|_{\mathrm{div}}^{2} \\
+\left\|z\left(u_{h}\right)-z_{h}\right\|_{0}^{2}+\left\|u-u_{h}\right\|_{U}^{2} \leq C \sum_{i=1}^{3} \eta_{i}^{2}
\end{gathered}
$$

where $\eta_{1}, \eta_{2}$, and $\eta_{3}$ are defined in Lemma 3.1, Lemma 3.2, and Lemma 3.4, respectively.

\section{A posteriori error estimates}

With the intermediate errors, we can decompose the errors as follows:

$$
\begin{aligned}
& \mathbf{p}-\mathbf{p}_{h}=\mathbf{p}-\mathbf{p}\left(u_{h}\right)+\mathbf{p}\left(u_{h}\right)-\mathbf{p}_{h}:=\epsilon_{1}+\varepsilon_{1}, \\
& y-y_{h}=y-y\left(u_{h}\right)+y\left(u_{h}\right)-y_{h}:=r_{1}+e_{1}, \\
& \mathbf{q}-\mathbf{q}_{h}=\mathbf{q}-\mathbf{q}\left(u_{h}\right)+\mathbf{q}\left(u_{h}\right)-\mathbf{q}_{h}:=\epsilon_{2}+\varepsilon_{2}, \\
& z-z_{h}=z-z\left(u_{h}\right)+z\left(u_{h}\right)-z_{h}:=r_{2}+e_{2} .
\end{aligned}
$$

By using the standard results of mixed finite element methods [26], we have the following results.

Lemma 4.1 There is a positive constant $C$ independent of h such that

$$
\begin{aligned}
& \left\|\epsilon_{1}\right\|_{\text {div }}+\left\|r_{1}\right\|_{0} \leq C\left\|u-u_{h}\right\|_{U}, \\
& \left\|\epsilon_{2}\right\|_{\text {div }}+\left\|r_{2}\right\|_{0} \leq C\left\|u-u_{h}\right\|_{u} .
\end{aligned}
$$

Proof It follows from (2.4)-(2.7) and (2.19)-(2.20) that we have the error equations:

$$
\begin{aligned}
& \left(A^{-1} \epsilon_{1}, \mathbf{v}\right)-\left(r_{1}, \operatorname{div} \mathbf{v}\right)=0, \quad \forall \mathbf{v} \in \mathbf{V}, \\
& \left(\operatorname{div} \epsilon_{1}, w\right)+\int_{\Omega} \int_{\Omega} G(s, t) r_{1}(s) w(t) d s d t=\left(B\left(u-u_{h}\right), w\right), \quad \forall w \in W, \\
& \left(A^{-1} \epsilon_{2}, \mathbf{v}\right)-\left(r_{2}, \operatorname{div} \mathbf{v}\right)=-\left(g_{1}^{\prime}(\mathbf{p})-g_{1}^{\prime}\left(\mathbf{p}\left(u_{h}\right)\right), \mathbf{v}\right), \quad \forall \mathbf{v} \in \mathbf{V}, \\
& \left(\operatorname{div} \epsilon_{2}, w\right)+\int_{\Omega} \int_{\Omega} G(s, t) r_{2}(t) w(s) d s d t=\left(g_{2}^{\prime}(y)-g_{2}^{\prime}\left(y\left(u_{h}\right)\right), w\right), \quad \forall w \in W .
\end{aligned}
$$

Choosing $\mathbf{v}=\epsilon_{1}$ and $w=r_{1}$ as the test functions and adding the two relations of (4.3)-(4.4), we have

$$
\left(A^{-1} \epsilon_{1}, \epsilon_{1}\right)+\int_{\Omega} \int_{\Omega} G(s, t) r_{1}(s) r_{1}(t) d s d t=\left(B\left(u-u_{h}\right), r_{1}\right) .
$$


Then, using the assumption on $A$ and (1.7), we obtain that

$$
\left\|\epsilon_{1}\right\|_{0}^{2}+\left\|r_{1}\right\|_{0}^{2} \leq C\left\|u-u_{h}\right\|_{0}^{2}+\delta\left\|r_{1}\right\|_{0}^{2}
$$

Now we choose $\mathbf{v}=\operatorname{div} \epsilon_{1}$ in equation (4.4), then we obtain

$$
\left(\operatorname{div} \epsilon_{1}, \operatorname{div} \epsilon_{1}\right)=\left(B\left(u-u_{h}\right), \operatorname{div} \epsilon_{1}\right)-\int_{\Omega} \int_{\Omega} G(s, t) r_{1}(s) \operatorname{div} \epsilon_{1}(t) d s d t
$$

Then, using the $\delta$-Cauchy inequality, we can find an estimate as follows:

$$
\begin{aligned}
\left\|\operatorname{div} \epsilon_{1}\right\|^{2} & \leq C\left(\left\|u-u_{h}\right\|^{2}+\left\|\int_{\Omega} G(s, t) r_{1}(s) d s\right\|^{2}\right)+\delta\left\|\operatorname{div} \epsilon_{1}\right\|^{2} \\
& \leq C\left(\left\|u-u_{h}\right\|^{2}+\left\|r_{1}\right\|^{2}\right)+\delta\left\|\operatorname{div} \epsilon_{1}\right\|^{2} .
\end{aligned}
$$

Thus,

$$
\left\|\operatorname{div} \epsilon_{1}\right\|^{2} \leq C\left(\left\|u-u_{h}\right\|^{2}+\left\|r_{1}\right\|^{2}\right) \leq C\left\|u-u_{h}\right\|^{2} .
$$

This implies (4.1).

Similarly, we choose $\mathbf{v}=\epsilon_{2}$ and $w=r_{2}$ as the test functions and add the two relations of (4.5)-(4.6), then we have

$$
\begin{aligned}
& \left(A^{-1} \epsilon_{2}, \epsilon_{2}\right)+\int_{\Omega} \int_{\Omega} G(s, t) r_{2}(t) r_{2}(s) d s d t \\
& \quad=\left(g_{2}^{\prime}(y)-g_{2}^{\prime}\left(y\left(u_{h}\right)\right), r_{2}\right)-\left(g_{1}^{\prime}(\mathbf{p})-g_{1}^{\prime}\left(\mathbf{p}\left(u_{h}\right)\right), \epsilon_{2}\right) .
\end{aligned}
$$

Then, using the assumption on $A$ and (1.7), we obtain that

$$
\left\|\epsilon_{2}\right\|_{0}^{2}+\left\|r_{2}\right\|_{0}^{2} \leq C\left(\left\|\epsilon_{1}\right\|_{0}^{2}+\left\|r_{1}\right\|_{0}^{2}\right)+\delta\left(\left\|\epsilon_{2}\right\|_{0}^{2}+\left\|r_{2}\right\|_{0}^{2}\right)
$$

Hence, we derive that

$$
\left\|\epsilon_{2}\right\|_{0}^{2}+\left\|r_{2}\right\|_{0}^{2} \leq C\left(\left\|\epsilon_{1}\right\|_{0}^{2}+\left\|r_{1}\right\|_{0}^{2}\right) \leq C\left\|u-u_{h}\right\|^{2}
$$

Now we choose $\mathbf{v}=\operatorname{div} \epsilon_{2}$ in equation (4.6), then we obtain

$$
\left(\operatorname{div} \epsilon_{2}, \operatorname{div} \epsilon_{2}\right)=\left(g_{2}^{\prime}(y)-g_{2}^{\prime}\left(y\left(u_{h}\right)\right), \operatorname{div} \epsilon_{2}\right)-\int_{\Omega} \int_{\Omega} G(s, t) r_{2}(t) \operatorname{div} \epsilon_{2}(s) d s d t
$$

Then, using the $\delta$-Cauchy inequality, we can find an estimate as follows:

$$
\left\|\operatorname{div} \epsilon_{2}\right\|^{2} \leq C\left(\left\|r_{1}\right\|^{2}+\left\|r_{2}\right\|^{2}\right)+\delta\left\|\operatorname{div} \epsilon_{2}\right\|^{2},
$$

and hence,

$$
\left\|\operatorname{div} \epsilon_{2}\right\|^{2} \leq C\left\|u-u_{h}\right\|^{2}
$$


Thus, (4.2) is proved by (4.13) and (4.16).

Hence, we combine Theorem 3.1 and Lemma 4.1 and use the triangle inequality to conclude the following.

Theorem 4.1 Let $(\mathbf{p}, y, \mathbf{q}, z, u) \in(\mathbf{V} \times W)^{2} \times U$ and $\left(\mathbf{p}_{h}, y_{h}, \mathbf{q}_{h}, z_{h}, u_{h}\right) \in\left(\mathbf{V}_{h} \times W_{h}\right)^{2} \times U_{h} b e$ the solutions of (2.4)-(2.8) and (2.14)-(2.18), respectively. Assume that $K_{h} \subset K$. In addition, assume that $\left.\left(J_{h}^{\prime}\left(u_{h}\right)\right)\right|_{s} \in H^{1}(s), \forall s \in \mathcal{T}_{h}\left(\Omega_{U}\right)$, and that there is a $v_{h} \in K_{h}$ such that

$$
\left.\mid J_{h}^{\prime}\left(u_{h}\right), v_{h}-u\right) \mid \leq C \sum_{s \in \mathcal{T}_{h}\left(\Omega_{U}\right)} h_{s}\left\|J_{h}^{\prime}\left(u_{h}\right)\right\|_{H^{1}(s)}\left\|u-u_{h}\right\|_{L^{2}(s)} .
$$

Then we have

$$
\left\|\mathbf{p}-\mathbf{p}_{h}\right\|_{\mathrm{div}}^{2}+\left\|y-y_{h}\right\|_{0}^{2}+\left\|\mathbf{q}-\mathbf{q}_{h}\right\|_{\mathrm{div}}^{2}+\left\|z-z_{h}\right\|_{0}^{2}+\left\|u-u_{h}\right\|_{U}^{2} \leq C \sum_{i=1}^{3} \eta_{i}^{2},
$$

where $\eta_{1}, \eta_{2}$, and $\eta_{3}$ are defined in Lemma 3.1, Lemma 3.2, and Lemma 3.4, respectively.

\section{Some applications}

In this section, we apply the previous results to two concrete optimal control problems.

Example 5.1 Consider the case $K=\{u \in U: u \geq 0\}$. Let $K_{h}=\left\{v \in U_{h}: v \geq 0\right\}$. Then it is easy to see that $K_{h} \subset K$. Let $v_{h}$ in Lemma 3.1 be such that $v_{h}=\Pi_{h} u$, where

$$
\left.\Pi_{h} w\right|_{x \in s}=\int_{s} w /|s|, \quad \forall w \in L^{2}\left(\Omega_{U}\right)
$$

where $|s|$ is the measure of the element $s$. Then $v_{h}=\Pi_{h} u \in K_{h}$, and

$$
\begin{aligned}
\left|\left(j^{\prime}\left(u_{h}\right)+B^{*} z_{h}, v_{h}-u\right)\right| & =\left|\left(j^{\prime}\left(u_{h}\right)+B^{*} z_{h}, \Pi_{h} u-u\right)\right| \\
& =\left|\left(j^{\prime}\left(u_{h}\right)+B^{*} z_{h}-\Pi_{h}\left(j^{\prime}\left(u_{h}\right)+B^{*} z_{h}\right), \Pi_{h}\left(u-u_{h}\right)-\left(u-u_{h}\right)\right)\right| \\
& \leq \sum_{s \in \mathcal{T}_{h}\left(\Omega_{U}\right)} h_{s}\left\|j^{\prime}\left(u_{h}\right)+B^{*} z_{h}\right\|_{H^{1}(s)}\left\|u-u_{h}\right\|_{L^{2}(s)} .
\end{aligned}
$$

Hence, condition (3.4) in Lemma 3.1 is satisfied. If all the conditions in Theorem 4.1 hold, then

$$
\left\|\mathbf{p}-\mathbf{p}_{h}\right\|_{\mathrm{div}}^{2}+\left\|y-y_{h}\right\|_{0}^{2}+\left\|\mathbf{q}-\mathbf{q}_{h}\right\|_{\mathrm{div}}^{2}+\left\|z-z_{h}\right\|_{0}^{2}+\left\|u-u_{h}\right\|_{U}^{2} \leq C \sum_{i=1}^{3} \eta_{i}^{2},
$$

where $\eta_{1}, \eta_{2}$, and $\eta_{3}$ are defined in Lemma 3.1, Lemma 3.2, and Lemma 3.4, respectively.

Example 5.2 Consider the case $K=\left\{u \in U: \int_{\Omega_{U}} u \geq 0\right\}$. Let $K_{h}=\left\{v \in U_{h}: \int_{\Omega_{U}} v \geq 0\right\}$. Then it is easy to see that $K_{h} \subset K$. Let $v_{h}$ in Lemma 3.1 be such that $v_{h}=\Pi_{h} u$, where $\Pi_{h}$ is defined as in Example 5.1. Then $v_{h}=\Pi_{h} u \in K_{h}$, and similarly as in Example 5.1,

$$
\left|\left(j^{\prime}\left(u_{h}\right)+B^{*} z_{h}, v_{h}-u\right)\right| \leq \sum_{s \in \mathcal{T}_{h}\left(\Omega_{U}\right)} h_{s}\left\|j^{\prime}\left(u_{h}\right)+B^{*} z_{h}\right\|_{H^{1}(s)}\left\|u-u_{h}\right\|_{L^{2}(s)} .
$$


Hence, condition (3.4) in Lemma 3.1 is satisfied. If all the conditions in Theorem 4.1 hold, then

$$
\left\|\mathbf{p}-\mathbf{p}_{h}\right\|_{\mathrm{div}}^{2}+\left\|y-y_{h}\right\|_{0}^{2}+\left\|\mathbf{q}-\mathbf{q}_{h}\right\|_{\mathrm{div}}^{2}+\left\|z-z_{h}\right\|_{0}^{2}+\left\|u-u_{h}\right\|_{U}^{2} \leq C \sum_{i=1}^{3} \eta_{i}^{2},
$$

where $\eta_{1}, \eta_{2}$, and $\eta_{3}$ are defined in Lemma 3.1, Lemma 3.2, and Lemma 3.4, respectively.

\section{Competing interests}

The authors declare that they have no competing interests.

\section{Authors' contributions}

ZL participated in the sequence alignment and drafted the manuscript.

\section{Author details}

${ }^{1}$ School of Mathematics and Statistics, Chongqing Three Gorges University, Chongqing, 404000, P.R. China. ${ }^{2}$ College of Civil Engineering and Mechanics, Xiangtan University, Xiangtan, 411105, P.R. China. ${ }^{3}$ Chongqing Wanzhou Senior Middle School, Chongqing, 404000, P.R. China.

\section{Acknowledgements}

The author express his thanks to the referees for their helpful suggestions, which led to improvements of the presentation. This work is supported by the National Science Foundation of China (11201510), Mathematics TianYuan Special Funds of the National Natural Science Foundation of China (11126329), China Postdoctoral Science Foundation funded project (2011M500968), Natural Science Foundation Project of CQ CSTC (cstc2012jjA00003), Natural Science Foundation of Chongqing Municipal Education Commission (KJ121113), and Science and Technology Project of Wanzhou District of Chongqing (2013030050).

Received: 18 March 2012 Accepted: 9 July 2013 Published: 29 July 2013

\section{References}

1. Falk, FS: Approximation of a class of optimal control problems with order of convergence estimates. J. Math. Anal. Appl. 44, 28-47 (1973)

2. Geveci, T: In the approximation of the solution of an optimal control problem governed by an elliptic equation. RAIRO. Anal. Numér. 13, 313-328 (1979)

3. Li, R, Liu, W, Ma, H, Tang, T: Adaptive finite element approximation for distributed convex optimal control problems. SIAM J. Control Optim. 41, 1321-1349 (2002)

4. Brunner, H, Yan, N: Finite element methods for optimal control problems governed by integral equations and integro-differential equations. Numer. Math. 101, 1-27 (2005)

5. Lions, JL: Optimal Control of Systems Governed by Partial Differential Equations. Springer, Berlin (1971)

6. Liu, W, Yan, N: A posteriori error estimates for control problems governed by nonlinear elliptic equations. Appl. Numer. Math. 47, 173-187 (2003)

7. Liu, W, Yan, N: A posteriori error estimates for control problems governed by Stokes equations. SIAM J. Numer. Anal. 40, 1850-1869 (2002)

8. Brunner, H, Yan, N: On global superconvergence of iterated collocation solutions to linear second-kind Volterra integral equations. J. Comput. Appl. Math. 67, 185-189 (2005)

9. Yan, N: Superconvergence analysis and a posteriori error estimates of a finite element method for an optimal control problem governed by integral equations. Appl. Math. 54, 267-283 (2009)

10. Chen, Y: Superconvergence of mixed finite element methods for optimal control problems. Math. Comput. 77, 1269-1291 (2008)

11. Chen, Y: Superconvergence of quadratic optimal control problems by triangular mixed finite elements. Int. J. Numer. Methods Eng. 75, 881-898 (2008)

12. Chen, Y, Dai, L, Lu, Z: Superconvergence of quadratic optimal control problems by triangular mixed finite elements. Adv. Appl. Math. Mech. 75, 881-898 (2009)

13. $L u, Z$, Chen, $Y: L^{\infty}$-error estimates of triangular mixed finite element methods for optimal control problem govern by semilinear elliptic equation. Numer. Anal. Appl. 12, 74-86 (2009)

14. Xing, $X$ Chen, $Y: L^{\infty}$-error estimates for general optimal control problem by mixed finite element methods. Int. J. Numer. Anal. Model. 5, 441-456 (2008)

15. Chen, Y, Liu, W: A posteriori error estimates for mixed finite element solutions of convex optimal control problems. J. Comput. Appl. Math. 211, 76-89 (2008)

16. Lu, Z, Chen, Y: A posteriori error estimates of triangular mixed finite element methods for semilinear optimal control problems. Adv. Appl. Math. Mech. 1, 242-256 (2009)

17. Kress, R: Linear Integral Equation, 2nd edn. Springer, New York (1999)

18. Zabreiko, PP, Koshelev, Al, Krasnosel'skii, MA, Mikhlin, SG, Rakovshchik, LS, Stet'senko, VY: Integral Equations-a Reference Text. Noordhoff, Groningen (1975)

19. Chen, Y, Liu, W: Posteriori error estimates for mixed finite elements of a quadratic optimal control problem. In: Recent Progress Comput. Appl. PDEs, vol. 2, pp. 123-134 (2002) 
20. Raviart, PA, Thomas, JM: A mixed finite element method for 2nd order elliptic problems. In: Mathematical Aspects of the Finite Element Method. Lecture Notes in Math., vol. 606, pp. 292-315. Springer, Berlin (1977)

21. Bonnans, JF: Second-order analysis for control constrained optimal control problems of semilinear elliptic systems. Appl. Math. Optim. 38, 303-325 (1998)

22. Babuska, I, Strouboulis, T: The Finite Element Method and Its Reliability. Oxford University Press, Oxford (2001)

23. Carstensen, C: A posteriori error estimate for the mixed finite element method. Math. Comput. 66, 465-476 (1997)

24. Arada, N, Casas, E, Tröltzsch, F: Error estimates for the numerical approximation of a semilinear elliptic control problem. Comput. Optim. Appl. 23, 201-229 (2002)

25. Verfürth, R: A Review of a Posteriori Error Estimation and Adaptive Mesh-Refinement Techniques. Wiley, New York (1996)

26. Brezzi, F, Fortin, M: Mixed and Hybrid Finite Element Methods. Springer, Berlin (1991)

doi:10.1186/1029-242X-2013-351

Cite this article as: Lu and Liu: A posteriori error estimates of mixed finite element methods for general optimal control problems governed by integro-differential equations. Journal of Inequalities and Applications 2013 2013:351.

\section{Submit your manuscript to a SpringerOpen ${ }^{0}$ journal and benefit from:}

- Convenient online submission

- Rigorous peer review

Immediate publication on acceptance

Open access: articles freely available online

- High visibility within the field

- Retaining the copyright to your article 